OPEN ACCESS

Edited by: Annarosa Arcangeli, University of Florence, Italy

Reviewed by:

Wei Wang,

Fourth Military Medical University,

China

Agnieszka Zdzisława Robaszkiewicz,

University of Łódź, Poland

*Correspondence:

Yonghua Xue

86976512@qq.com

Wei Xu

XW2107@shtrhospital.com

${ }^{\dagger}$ These authors have contributed equally to this work

Specialty section:

This article was submitted to Pharmacology of Anti-Cancer Drugs, a section of the journal

Frontiers in Pharmacology

Received: 01 March 2020

Accepted: 10 August 2020

Published: 26 August 2020

Citation:

Gu Z, Li Z, Xu R, Zhu X, Hu R, Xue Y and Xu W (2020) miR-16-5p

Suppresses Progression and Invasion of Osteosarcoma

via Targeting at Smad3.

Front. Pharmacol. 11:1324. doi: 10.3389/fphar.2020.01324

\section{miR-16-5p Suppresses Progression and Invasion of Osteosarcoma via Targeting at Smad3}

\author{
Zhijian $\mathrm{Gu}^{1 \dagger}$, Zhikun $\mathrm{Li}^{1 \dagger}$, Ruijun $\mathrm{Xu}{ }^{1}$, Xiaodong $\mathrm{Zhu}{ }^{1}$, Ruixi $\mathrm{Hu}^{1}$, Yonghua Xue ${ }^{2 *}$ \\ and Wei $\mathrm{Xu}{ }^{\text {* }}$ \\ 1 Department of Orthopedics, Tongren Hospital, School of Medicine, Shanghai Jiao Tong University, Shanghai, China, \\ ${ }^{2}$ Department of Neurosurgery, Putuo District Central Hospital, Shanghai University of Traditional Chinese Medicine, \\ Shanghai, China
}

Background: MicroRNAs are known to regulate carcinogenesis of osteosarcoma. Although, miR-16-5p is known to exert inhibitory effects on several forms of cancers, its effects on the growth and invasion of osteosarcoma have not been studied.

Methods: We collected human osteosarcoma specimens and adjacent tissues to detect the expression of miR-16-5p by real-time polymerase chain reaction, immunoblotting, and immunohistochemistry. The proliferation, migration, and invasion of MG63 and HOS cells following miR-16-5p overexpression and inhibition were detected with cell counting kit-8, wound healing assay, and Transwell assay, respectively. An expression vector carrying a mutated 3'-untranslated region of mothers against decapentaplegic homolog 3 (Smad3) was constructed.

Results: The results showed that miR-16-5p expression was downregulated in osteosarcoma tissues and cells as compared with adjacent counterparts, while Smad3 was overexpressed in osteosarcoma cells. The overexpression of miR-16-5p resulted in the inhibition of the proliferation, migration, and invasion of osteosarcoma cells and enhanced the therapeutic effect of cisplatin. These effects were attenuated with miR-16$5 p$ expression inhibition. In cells transfected with miR-16-5p mimic, Smad3 expression decreased, while this effect was absent in the cells carrying mutated Smad3.

Conclusions: Therefore, miR-16-5p inhibits the growth and invasion of osteosarcoma by targeting Smad3.

Keywords: osteosarcoma, miR-16-5p, invasion, Smad3, proliferation

\section{HIGHLIGHTS}

This study firstly investigated that miR-16-5p inhibited the proliferation, migration, and invasion of osteosarcoma via suppressing the expression of Smad3, meaning that miR-16-5p may be regarded as a potential target in the treatment of osteosarcoma. 


\section{INTRODUCTION}

Osteosarcoma is regarded as the most common primary malignant mesenchymal tumor of bones in both adults and adolescents (Yang and Zhang, 2013). Although many novel anti-cancer drugs or molecular targeted therapies have been developed over last few decades, the 5-year survival rate of patients with osteosarcoma is still around 60\% (Ritter and Bielack, 2010). However, the molecular mechanisms underlying carcinogenesis of osteosarcoma remain unknown and may involve mutations at genomic, transcriptomic, and proteomic levels (Scotlandi et al., 1996).

Epigenetic events can cause tumorigenesis (Foley et al., 2015; Chano et al., 2016; Li et al., 2018). As small non-coding RNAs, micro-RNAs (miRNAs) are short single-stranded ribonucleic acid (usually contain 17-25 nucleotides) that may complementarily pair with the $3^{\prime}$-untranslated regions $\left(3^{\prime}\right.$ UTRs) of their target messenger RNAs (mRNAs) and induce gene-silencing effects through the degradation of their targets. Studies have demonstrated that miRNA may modulate the chemotherapeutic sensitivity of osteosarcoma cells (Meng et al., 2017; Song et al., 2017; Wang et al., 2017). miR-16-5p has been demonstrated as an vital component of the intracellular microRNA regulatory network. A remarkably inhibitory effect of miR16-5p on the growth and invasion of cancer, including breast cancer, gastric cancer, osteosarcoma, and chordoma, has been reported previously (Rinnerthaler et al., 2016; Qu et al., 2017; Sang et al., 2017; Zhu et al., 2018; Zhang et al., 2018). Exosomeassociated micro-RNA panels were recently thought to predict drug resistance in patients with multiple myeloma, wherein the expression of miR-16-5p was found to be downregulated in patients resistant to bortezomib (Zhang et al., 2016). miR-16-5p is shown to inhibit gastric cancer, breast cancer, and chordoma in previous researches (Zhang et al., 2015; Rinnerthaler et al., 2016; Zhu et al., 2018; Zhang et al., 2018). However, the function of miR-16-5p in the progression and chemosensitivity of osteosarcoma still remains unknown.

Smad3, as an important transporter in the transforming growth factor $\beta$ (TGF- $\beta$ ) signaling pathway, can transports the TGF- $\beta$ signal from the cell membrane into the nucleus, so that the TGF- $\beta$ can combine with relevant nuclear factors to regulate the expression of target genes, thereby, for controlling cell proliferation. Abnormal expression and function of $\operatorname{smad} 3$ will lead to dysfunction of proliferation, differentiation, migration, and apoptosis of cell, and eventually can trigger the occurrence, progression, and metastasis of cancer (Tang et al., 2017; Rypens et al., 2020). It was revealed that osteosarcoma cells could release TGF- $\beta$, which can be suppressed by inhibitors of TGF- $\beta$ type I receptor and knockdown of Smad3. Moreover, significant expressions of CD42b, TF, TGF- $\beta$, Smad2/3, and $\mathrm{p}$-Smad2/3 were also detected in a biopsy sample from an osteosarcoma patient compared with control. Collectively, these finding suggested that the interaction between osteosarcoma cells and platelets, via thrombin and TGF- $\beta$, results in a continuous cycle, and that anti-TGF- $\beta$ or anti-Smad 3 therapy could be a promising tool for disease treatment (Saito et al., 2018).

In the present study, we reveal the significant decrease in the expression level of miR-16-5p in osteosarcoma cells as compared with normal cells. Upregulation in miR-16-5p expression significantly inhibits the proliferation, migration, and invasion of osteosarcoma cells by silencing mothers against decapentaplegic homolog $3(\operatorname{Smad} 3)$ expression.

\section{METHODS AND MATERIALS}

\section{Tissue Samples of Patients and Cell Culture}

The studies involving human participants were reviewed and approved by Institutional Review Board of Tongren Hospital (No. 2019-015). The patients provided their written informed consent to participate in this study. The informed consent in accordance with the Declaration of Helsinki were signed and obtained from all donors.

In this study, osteosarcoma specimens were obtained from 40 patients that underwent surgical resections in Orthopedics of Tongren Hospital from June 2012 to October 2014. The tissues were snap-frozen in liquid nitrogen and stored at $-80^{\circ} \mathrm{C}$. Adjacent soft tissues were obtained from the same patients. The clinical and pathological materials of patients were displayed in Table 1. A human osteoblast precursor cell line, hFOB1.19, and osteosarcoma cell lines, MG63, SaOS2, HOS, and U2OS, were provided by the American Type Culture Collection (ATCC). Dulbecco's modified Eagle's medium (DMEM) added with $10 \%$ fetal bovine serum (FBS) were used to culture cells in an atmosphere at $5 \% \mathrm{CO}_{2}$ and $37^{\circ} \mathrm{C}$.

\section{Cell Transfection}

The siRNA targeting Smad3, scrambled negative control (NC) siRNA, miR-16-5p mimic, miR-NC, miR-16-5p inhibitor, and NC inhibitor were obtained from GenePharma (Suzhou, Jiangsu, China). MG63 and HOS cells were transfected with siRNA, miR16-5p mimic, miR-NC, miR-16-5p inhibitor, and NC inhibitor

TABLE 1 | Clinical-pathological materials of patients with osteosarcoma.

\begin{tabular}{|c|c|c|c|c|}
\hline \multirow{2}{*}{$\begin{array}{l}\text { Clinical and } \\
\text { pathological parameters }\end{array}$} & \multirow[t]{2}{*}{ Total (n) } & \multicolumn{3}{|c|}{ miR-16-5p expression } \\
\hline & & High & Low & $p$-value \\
\hline Total & 40 & 23 & 17 & \\
\hline Gender & & & & $0.5473(N S)$ \\
\hline Male & 21 & 13 & 8 & \\
\hline Female & 19 & 10 & 9 & \\
\hline Age, years & & & & 0.6798 (NS) \\
\hline$<50$ & 15 & 8 & 7 & \\
\hline$>50$ & 25 & 15 & 10 & \\
\hline Histological stage & & & & $0.049^{*}$ \\
\hline Well differentiated & 19 & 14 & 5 & \\
\hline Poor differentiated & 21 & 9 & 12 & \\
\hline Tumor diameter, $\mathbf{c m}$ & & & & $0.001^{*}$ \\
\hline$\leq 5$ & 19 & 16 & 4 & \\
\hline$>5$ & 29 & 7 & 13 & \\
\hline TNM stage & & & & $0.002^{\star}$ \\
\hline | and || & 23 & 18 & 5 & \\
\hline III and IV & 17 & 5 & 12 & \\
\hline
\end{tabular}

NS, no significant.

*means $p<0.05$. 
using Lipofectamine 3000 (Invitrogen), as per the manufacturer's instructions. The final concentrations of miRNA mimic and siRNA were 100 and $20 \mathrm{nM}$, respectively.

The sequences were as follows:

miR-16-5p mimic: 5'-UAGCAGCACGUAAAUAUUGGCG-3'

miR-16-5p inhibitor: 5'-CACCAAUAUUUACGUGCUG CUA-3'

miR-NC: 5'-UUCUCCGAACGUGUCACGUTT-3'

Smad3 siRNA: 5'-CCGCAU GAGCUUCGUCAAATT-3'

\section{Cell Viability Assay}

The proliferation of cells was evaluated using cell-counting kit-8 (CCK-8). Briefly, HOS or MG63 cells transfected with miR-16-5p mimic, miR-NC, miR-16-5p inhibitor, or NC inhibitor were seeded in 96-well plates at $1 \times 10^{4}$ cells/well and incubated for $24,48,72$, and $96 \mathrm{~h}$. At each time point, the media were discarded and replaced with fresh serum-free media containing CCK- 8 solution at a final concentration of $10 \%(\mathrm{v} / \mathrm{v})$. The plates were incubated in the dark at $37^{\circ} \mathrm{C}$ and $5 \% \mathrm{CO}_{2}$ atmosphere for $2 \mathrm{~h}$. Then, the ODs were measured using a microplate reader at $450 \mathrm{~nm}$. All results were obtained from three independent experiments.

\section{Total Messenger RNA Extraction and Real-Time Polymerase Chain Reaction (RT-PCR) Assay}

Total mRNAs were extracted using the Qiagen RNeasy ${ }^{\circledR}$ Mini Kit (Valencia, CA, USA) according to the instructions provided by the manufacturer before reverse transcription into complementary DNA (cDNA). Real-time PCR (RT-PCR) was conducted with SYBR ${ }^{\circledR}$ Premix Ex TaqTM Kit on Applied Biosystems 7500 Real-Time PCR instrument. The U6 small nuclear RNA and glyceraldehyde-3-phosphate dehydrogenase $(G A P D H)$ mRNA were used as internal controls for miR-16-5p and Smad3 mRNA, respectively. The sequences of primers for RT-PCR were as follows:

Smad3 forward primer,

5'-GTCTGCAAGATCCCACCAG-3' and reverse primer,

5'-AGCCCTGGTTGACCGACT-3';

GAPDH forward primer,

5'-ACCCAGAAGACTGTGGATGG-3' and reverse primer,

5'-CACATTGGGGGTAGGAACAC-3';

U6 small nuclear RNA forward primer, 5'-CTCGCTTCG

GCAGCACA-3' and reverse primer, 5'-AACGCTTCACGAATTTGCGT-3'.

The experiments were repeated thrice independently.

\section{Western Blot Analysis}

Cells were harvested and lysed with radioimmunoprecipitation assay (RIPA) buffer on ice for the isolation of total proteins. Cells were centrifuged at $10,000 \times g$ for $10 \mathrm{~min}$ and the supernatants were carefully collected and used as total protein after detection of protein concentration using bicinchoninic acid (BCA) kit (Thermo Fisher Scientific). The cell lysate was separated using electrophoresis and the separated protein bands were transferred onto polyvinylidene difluoride (PVDF) membranes, followed by blocked using $5 \%$ fat-free milk for $1 \mathrm{~h}$. Then, the membranes were incubated with primary antibodies at $4^{\circ} \mathrm{C}$ overnight. The next day, membranes were then treated with a fluorescent second antibody for $1 \mathrm{~h}$ and visualized with a chemiluminescence system (Millipore). The experiments were repeated thrice.

\section{Dual-Luciferase Reporter Assay}

The cDNA of the wild-type or mutated Smad3 containing the binding site of miR-16-5p at the $3^{\prime}$-UTR were synthesized by GenScript (Nanjing, Jiangsu, China) and integrated into pmirGLO luciferase reporter vector (Promega, Madison, WI, USA). MG63 and HOS cells were seeded in 96-well plates and incubated for $24 \mathrm{~h}$. Cells were transfected with the wild-type or mutated luciferase reporter vector along with miR-16-5p mimic, miR-NC, miR-16-5p inhibitor, or NC inhibitor for $48 \mathrm{~h}$. Luciferase activity was detected using a Dual-Luciferase Assay Kit (Promega, CA, USA).

\section{Wound Healing Assay}

A total of $1 \times 10^{5} \mathrm{MG} 63$ cells/well were seeded in six-well plates and allowed to attach. Cells were transfected with miR-16-5p mimic or miR-NC and cultured until $90 \%$ confluence. A scratch was produced with a pipette tip in the middle of every well and the healing rate was observed at 24 and $48 \mathrm{~h}$. The experiments were repeated thrice.

\section{Transwell Invasion Assays}

We used 24-well Transwell inserts (Corning Costar, Cambridge, MA, USA) to investigate the invasive capability of osteosarcoma cells. A total of $5 \times 10^{4}$ MG63 cells transfected with miR-16-5p mimic, miR-NC, miR-16-5p inhibitor, or NC inhibitor in $200 \mu \mathrm{l}$ of serum-free media were seeded onto the upper chamber of Transwell inserts and $500 \mu \mathrm{l}$ of media containing 10\% FBS used as a chemoattractant were added into the lower chambers. After $48 \mathrm{~h}$, the cells in the upper chamber were removed with a swab and the inferior surface of the membrane was stained with $0.1 \%$ crystal violet after treatment with $4 \%$ paraformaldehyde. Positively stained cells were counted in five fields per well under a microscope. Experiments were repeated thrice.

\section{Immunohistochemistry Assays}

Human osteosarcoma samples or adjacent tissues were fixed with formalin, embedded into paraffin, and sectioned as $5 \mu \mathrm{m}$ slices. After deparaffinization and dehydration in graded ethanol solutions, tissue sections were incubated in 3\% hydrogen peroxide solution for blocking endogenous peroxidases. The tissues were treated with a primary antibody against Smad3 at $4^{\circ} \mathrm{C}$ overnight. Following incubation, the samples were treated with a horseradish peroxidase (HRP)-conjugated secondary antibody at room temperature for $2 \mathrm{~h}$. The sections were washed, counterstained with diaminobenzidine (DAB), and visualized under a microscope. 


\section{Terminal Deoxynucleotidyl Transferase- Mediated Deoxyuridine Triphosphate- Biotin Nick End Labeling Staining}

The apoptotic level of tumor cells in tumor tissue were evaluated by terminal deoxynucleotidyl transferase (TdT)-mediated deoxyuridine triphosphate (dUTP)-biotin nick end labeling (TUNEL) using an In Situ Cell Death Detection Kit (Roche, Basel, Switzerland) according to the manufacturer's instructions. The tumor tissue sections were deparaffinized and rehydrated in an alcohol gradient. After washing with phosphate buffered saline (PBS), the sections were stained with the TUNEL reaction mixture, followed counterstaining with $4^{\prime}, 6$ diamidino-2-phenylindole (DAPI) $(1 \mu \mathrm{g} / \mathrm{ml})$. The results were visualized by green fluorescence was visualized in cells under a fluorescence microscope (Olympus, Beijing, China).

\section{Statistical Analysis}

All data were recorded as mean \pm standard deviation (SD) from at least three independent experiments. Student's $t$-test or chi- square test was used to analyze results using SPSS 21.0 software (Chicago, IL, USA). A value of $\mathrm{p}<0.05$ indicated significant difference.

\section{RESULTS}

\section{miR-16-5p Expression Is Downregulated in Osteosarcoma}

We evaluated the expression level of miR-16-5p in osteosarcoma and adjacent tissues using RT-PCR and found it to be significantly downregulated in osteosarcoma tissues as compared with adjacent tissues (Figure 1A). Retrospective Kaplan-Meier analysis of clinical samples revealed that the patients with high expression of miR-16-5p showed significantly longer survival than those with low expression of miR-16-5p, as analyzed with log-rank test ( $\mathrm{p}<$ 0.01 , Figure 1B). These results suggest that miR-16-5p expression inversely correlated with overall survival of patients. We evaluated
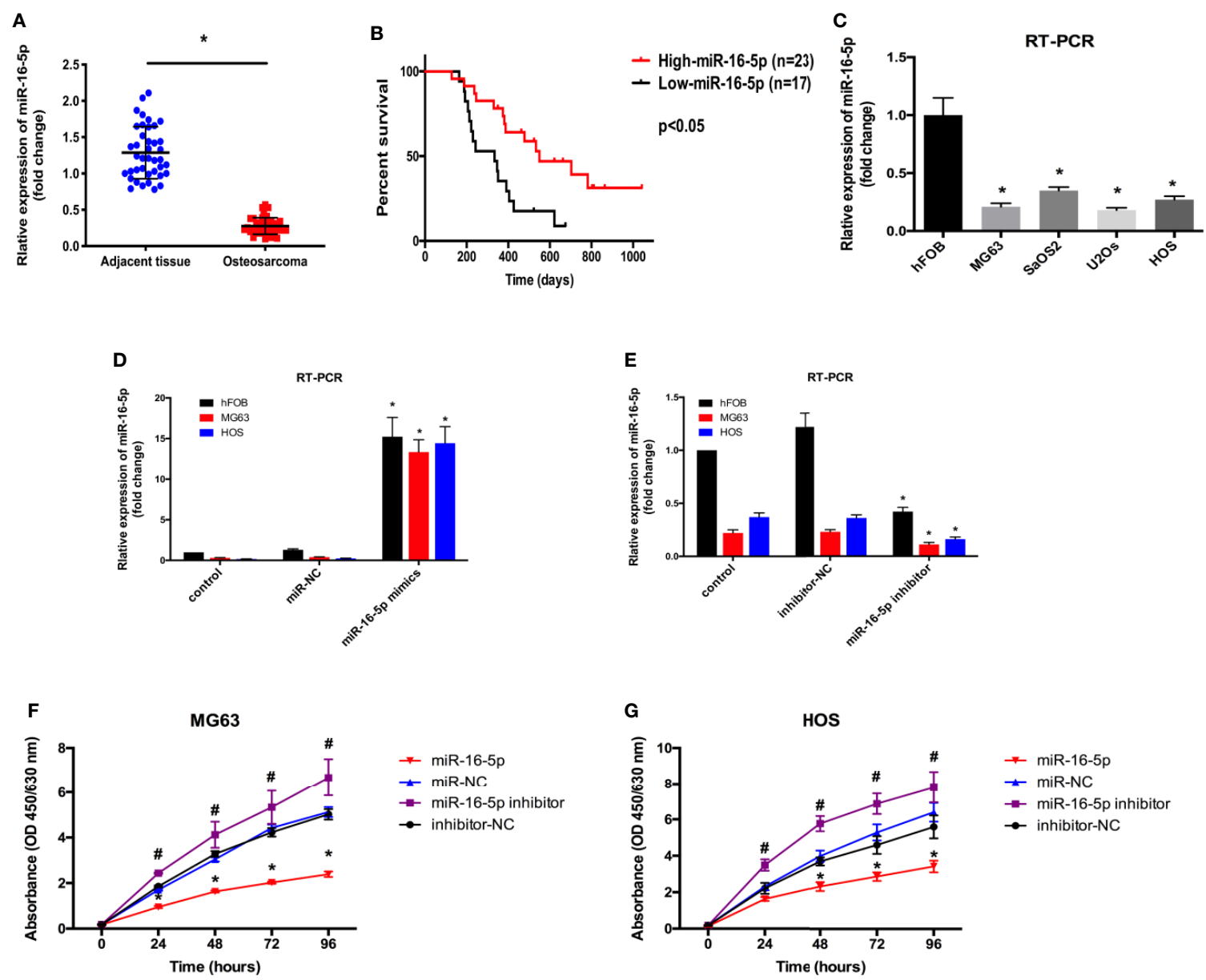

FIGURE 1 | The expression level of miR-16-5p in osteosarcoma and normal cells. (A, C) miR-16-5ps in human osteosarcoma and adjacent tissue samples and cell lines were detected by real-time PCR (RT-PCR). (B) Kaplan-Meier survival rates for overall survival (OS) patients with high ( $n=23)$ and low $(n=17)$ miR-16-5p expression. (D, E) miR-16-5p in cells transfected with mimics, inhibitor, and negative control were detected by RT-PCR. (F, G) the cell proliferation was evaluated by CCK-8. *indicates $p<0.05$. 
the expression of miR-16-5p in osteosarcoma cell lines and a human osteoblast cell line. Consistent with the observations reported in tissues, the level of miR-16-5p in hFOB1.19 cells was remarkable higher than that in MG63, SaOS2, U2OS, and HOS cells (Figure 1C). To evaluate the effects of transfection, miR-16$5 p$ expression level was detected in MG63 and HOS cells transfected with miR-16-5p mimic, miR-NC, miR-16-5p inhibitor, and NC inhibitor with RT-PCR. As a result, miR-16$5 \mathrm{p}$ was successfully overexpressed and repressed after transfection with mimic and inhibitor, respectively (Figures 1D, E). The proliferation of the cells transfected with miR-16-5p mimic, miR-NC, miR-16-5p inhibitor, or NC inhibitor was measured with CCK-8. Overexpression of miR-16-5p following transfection of miR-16-5p mimic resulted in the suppression of MG63 and HOS cell proliferation. On the contrary, the inhibition of miR-16$5 p$ expression following transfection with miR-16-5p inhibitor greatly promoted the proliferation of osteosarcoma cells (Figures 1F, G).

\section{miR-16-5p Inhibits the Migration and Invasion of Osteosarcoma Cells}

Wound healing assay was performed to investigate the effect of miR-16-5p expression on the migration of MG63 cells transfected with miR-NC, miR-16-5p mimic, miR-16-5p inhibitor, or NC inhibitor. After 24 and $48 \mathrm{~h}$ of incubation, the percentage of wound closure was evaluated. As a result, the wound in the group transfected with miR-16-5p mimic was remarkably wider than that in the control group transfected with miR-NC $(\mathrm{p}<0.05)$. The width of wound in miR-16-5p inhibitor-transfected cells was significantly smaller than that reported in control cells transfected with NC inhibitor ( $p<0.05$, Figures 2A, B). Thus, the upregulation of miR-16-5p expression inhibited the migration of osteosarcoma cells. Transwell invasion assay showed that the number of cells stained positive with crystal violet was lower in the group transfected with miR-16-5p $(49 \pm 8)$ than in the control group transfected with miR-NC $(137 \pm 21)(\mathrm{p}<0.05$, Figures $2 \mathrm{C}$, D). However, the number of positively stained cells in the group transfected with miR-16-5p inhibitor $(281 \pm 36)$ was significantly higher than that in the control group transfected with $\mathrm{NC}$ inhibitor $(142 \pm 28)(p<0.05$, Figures 2C, D). Therefore, the overexpression of miR-16-5p significantly decreased the invasion of osteosarcoma cells. Overall, miR-16-5p expression inhibited the migration and invasion of osteosarcoma cells and promote the apoptosis of osteosarcoma cells in vitro.

\section{miR-16-5p Targets Smad3 in Osteosarcoma}

Results of RT-PCR analysis showed that the mRNA expression of Smad3 was upregulated in MG63, SaOS2, U2OS, and HOS cells at different levels as compared with that in hFOB1.19 cells (Figure 3A). Immunoblot assay revealed the increase in the expression of Smad3 in osteosarcoma cell lines (Figures 3B, C). The expression of Smad3 in human osteosarcoma specimens was detected with immunohistochemistry. Smad3 was overexpressed in the
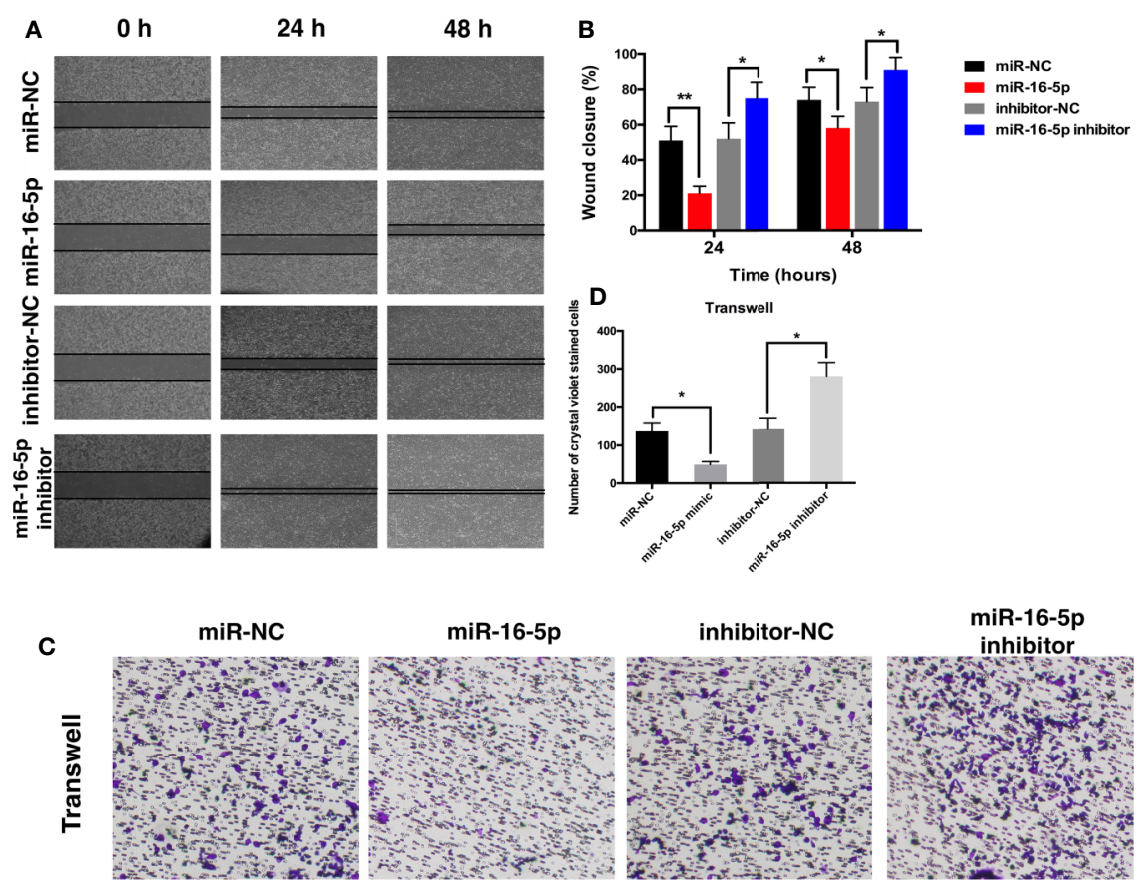

FIGURE 2 | Migration and invasion of osteosarcoma cells were inhibited by miR-16-5p. (A, B) wound-healing assays were performed using MG63 cells transfected with miR-16-5p mimics, miR-NC, miR-16-5p inhibitor, or inhibitor-NC for evaluating cells migration, and percentages of closure at 24 and 48 h were recorded. (C), D) Transwell assays and crystal violet staining were performed to detect the invasive capability of MG63 cells transfected with miR-NC, miR-16-5p mimics, miR-16-5p inhibitor, and inhibitor-NC, and positive stained cells was calculated. *indicates $p<0.05$. 

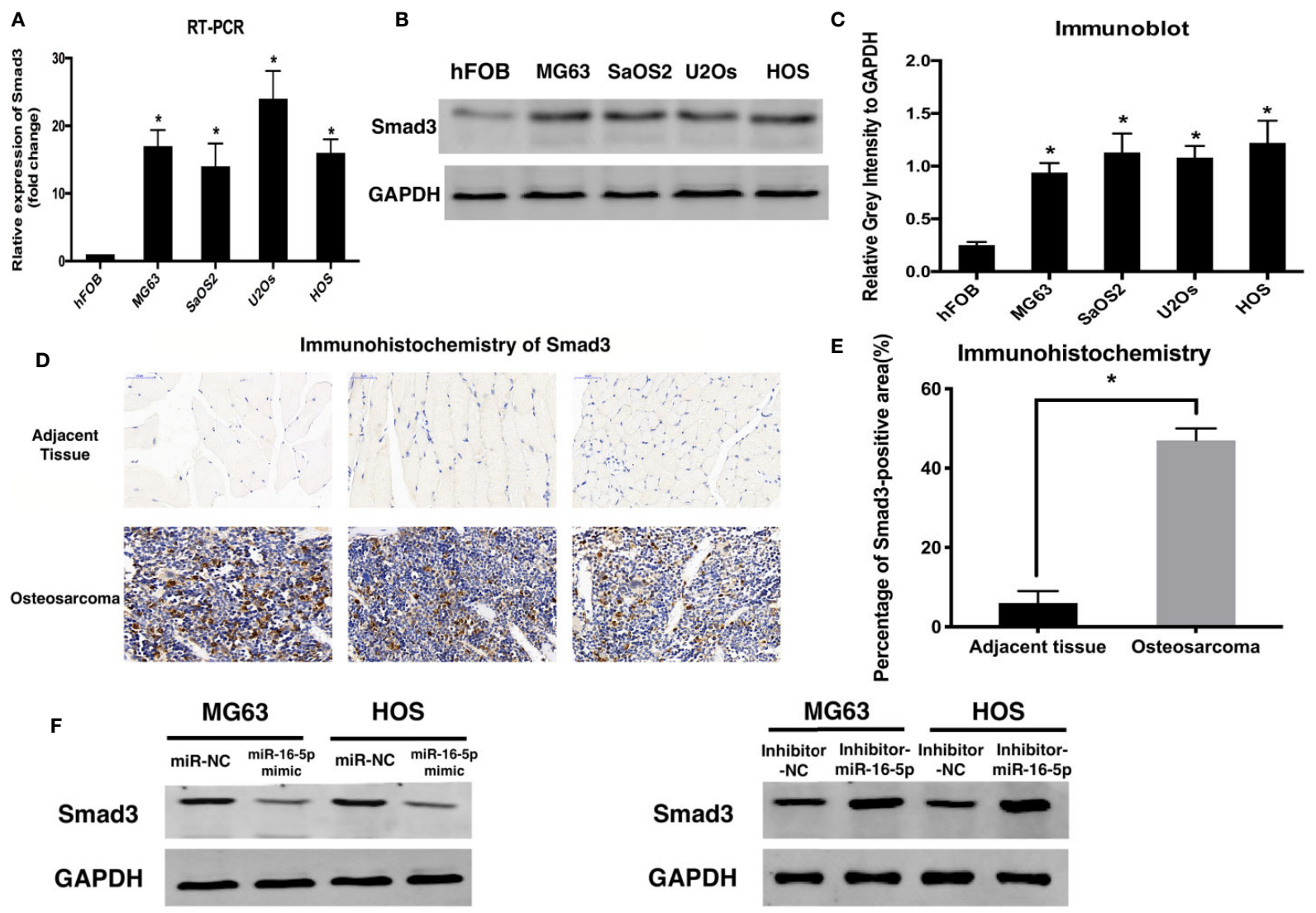

E
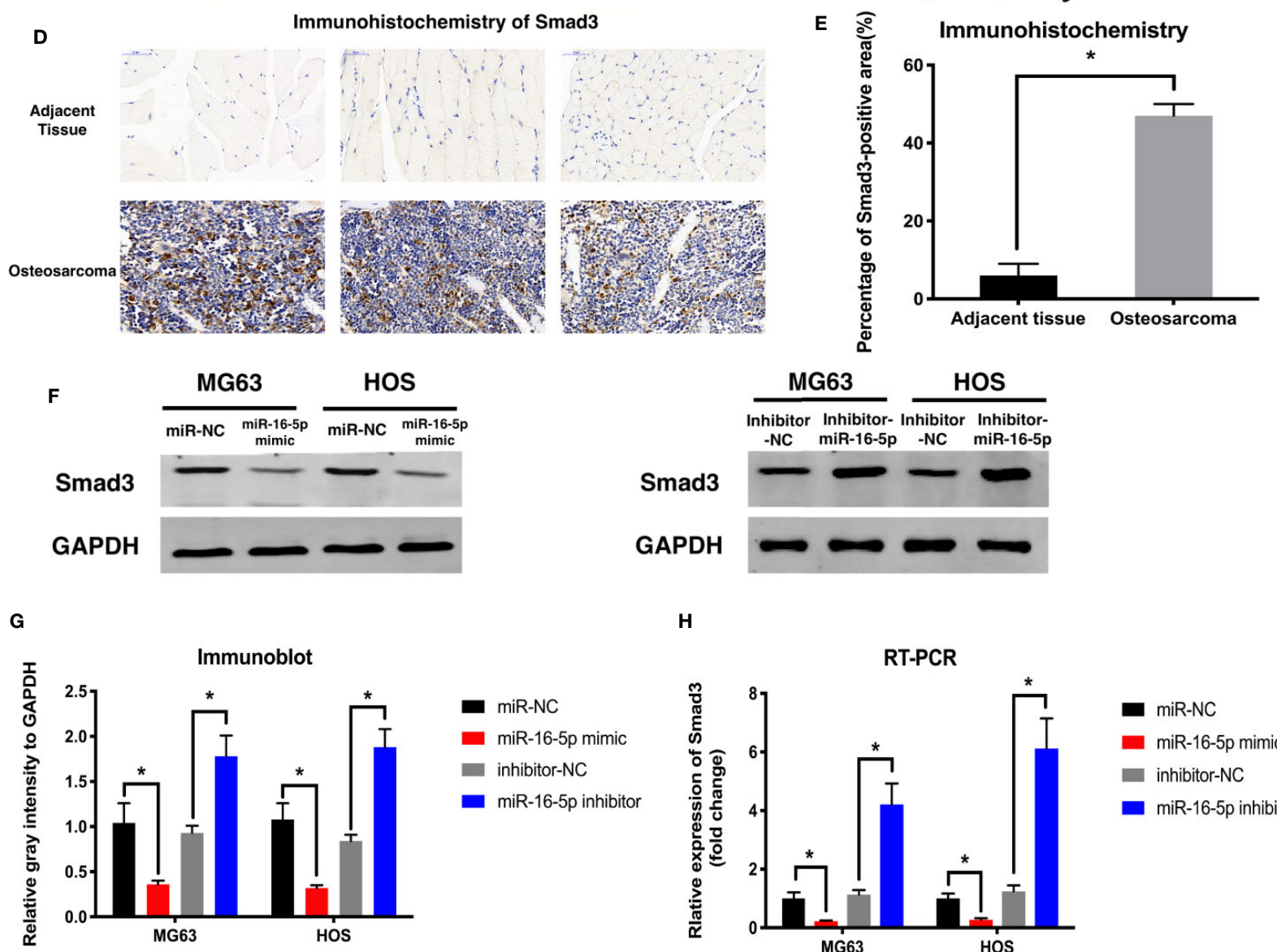

H

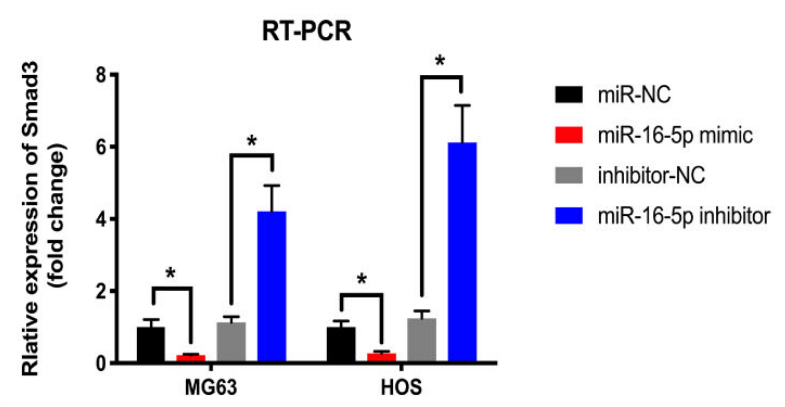

FIGURE 3 | Smad3 was regulated by miR-16-5p in osteosarcoma. (A) Real-time PCR (RT-PCR) and (B, C) immunoblot were performed to detect the expression of Smad3 in human osteoblasts and osteosarcoma cell lines. (D, E) Smad3 was analyzed by immunohistochemistry assays in human osteosarcoma and adjacent tissue. Smad3 in MG63 and HOS cells transfected with miR-16-5p mimics or inhibitor were detected by immunoblot (F, G) and RT-PCR (H). *indicates $p<0.05$.

osteosarcoma tissue as compared with the adjacent tissue (Figures 3D, E). We evaluated Smad3 expression in osteosarcoma cells transfected with miR-16-5p mimic, miR-NC, miR-16-5p inhibitor, or NC inhibitor with western blotting. The overexpression of miR-16-5p resulted in the suppression of Smad3 expression in osteosarcoma cells and this suppressive effect was attenuated following downregulation of miR-16-5p expression (Figures 3F, G). The results of RT-PCR showed that the mRNA expression of Smad3 decreased in MG63 and HOS cells transfected with miR-16-5p mimic as compared with that in control cells. The transfection of miR-16-5p inhibitor remarkably suppressed the expression of Smad3 in MG63 and HOS cells (Figure 3H). Therefore, miR-16-5p targeted Smad3 mRNA in MG63 and HOS cells, and this effect was abrogated following miR-16-5p inhibition.

\section{Overexpression of miR-16-5p Enhances the Susceptibility of Osteosarcoma Cells to Cisplatin}

To investigate the effect of miR-16-5p on the chemoresistance of osteosarcoma, MG63 and HOS cells transfected with miR-16-5p 
mimic, miR-NC, miR-16-5p inhibitor, and NC inhibitor were cultured in the presence of different concentrations of cisplatin for $48 \mathrm{~h}$. Cell viability was detected with CCK-8. As a result, the increase in miR-16-5p expression significantly enhanced the antitumor effects of cisplatin as compared with the control cells, while the inhibition of miR-16-5p expression promoted the resistance of MG63 and HOS cells to cisplatin treatment (Figures 4A, B) at 24, 48, 72, and 96 h of incubation (Figures 4C, D). Furthermore, osteosarcoma cells were incubated with cisplatin for $48 \mathrm{~h}$, and the expression of Smad3 mRNA and miR-16-5p was detected with RT-PCR. As a result, we found that the expression of miR-16-5p was downregulated, while that of Smad3 was upregulated.

\section{miR-16-5p Directly Targets Smad3}

To evaluate the effect of Smad3 targeting of miR-16-5p on the growth and chemoresistance of osteosarcoma, the vector expressing the mutated $3^{\prime}$-UTR of Smad3 was transfected into MG63 and HOS cells along with miR-16-5p mimic or inhibitor (Figure 5A). According to the results of cell viability assay, the proliferation of the cells expressing wild-type Smad3 and miR16-5p mimic was remarkably inhibited as compared with that of the control cells $(p<0.05)$. However, the inhibitory effect of miR16-5p was completely attenuated following mutation of Smad3 ( $p>0.05$, Figure 5B). The transcriptional activity of Smad3 in MG63 and HOS cells was measured with a dual-luciferase reporter assay. The luciferase activity was significantly lower in the cells transfected with the wild-type Smad3 and miR-16-5p mimic than in those transfected with the wild-type Smad3 and miR-NC (MG63: 1 versus $0.23 \pm 0.04$, HOS: 1 versus $0.18 \pm 0.02$, $\mathrm{p}<0.05)$. No reduction in luciferase activity was observed in the cells expressing the mutated $S \operatorname{mad} 3$ and miR-16-5p mimic (MG63: $0.97 \pm 0.11$ versus $1.07 \pm 0.08$; HOS: $1.04 \pm 0.13$ versus $1.02 \pm 0.10, \mathrm{p}>0.05)$. Therefore, overexpression of miR-16-5p resulted in a decrease in the transcriptional activity of Smad3 in MG63 and HOS cells, while this effect was attenuated in the cells expressing mutated Smad3 (Figure 5C). We investigated whether miR-16-5p decreases the expression of Smad3 protein by silencing the expression of Smad3 with the transfection of small-interfering RNA (siRNA)-Smad3. As a result, Smad3 expression was inhibited by miR-16-5p transfection in wildtype cells. The inhibitory effect of miR-16-5p on Smad3 expression was associated with the effect of siRNA-Smad3 (Figure 5D).

\section{DISCUSSION}

Many studies have evaluated the functions of miRNAs on the physiological and pathological behaviors of cells, including osteosarcoma cells (Shen et al., 2018; Xie et al., 2018). miRNAs not only regulate the carcinogenesis and metastasis of osteosarcoma cells but also influence their sensitivity to chemotherapy. miR-199a-3p was demonstrated to regulate drug resistance by targeting adenylate kinase 4 (AK4) (Lei et al., 2018). In addition, miR-140 expression was shown to overcome the chemoresistance of both osteosarcoma and colon cancer cells (Song et al., 2009). At present, very few studies have evaluated the function of miR-16-5p, and miR-16-5p may serve as a biomarker of early-stage rheumatoid arthritis, (Dunaeva

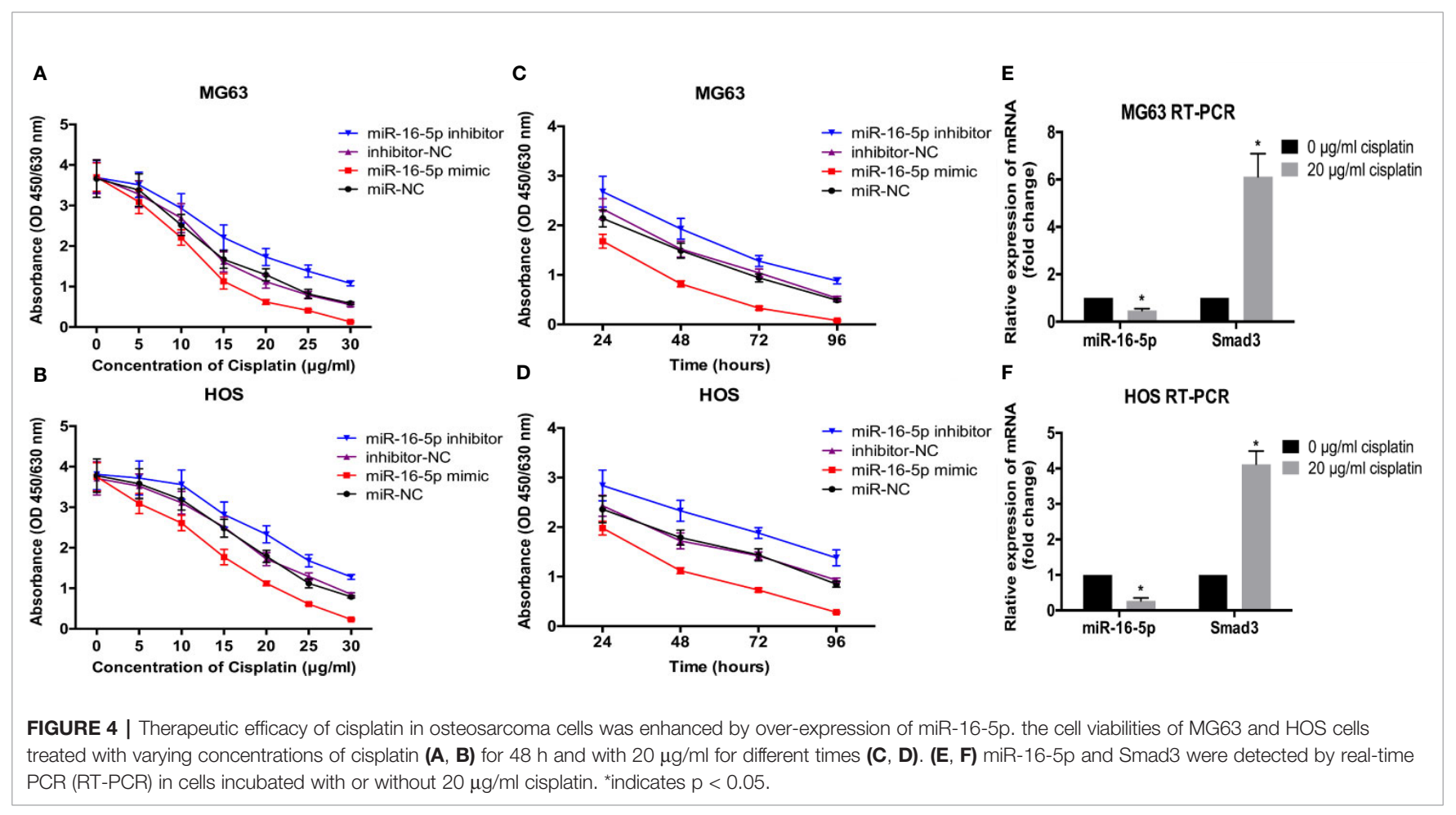


A

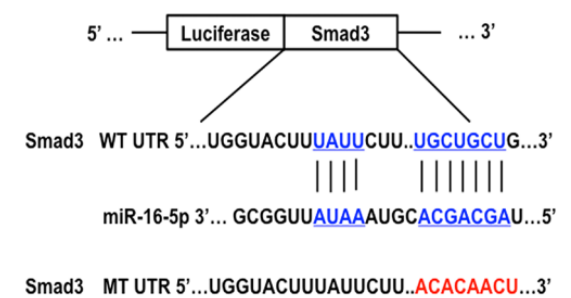

B

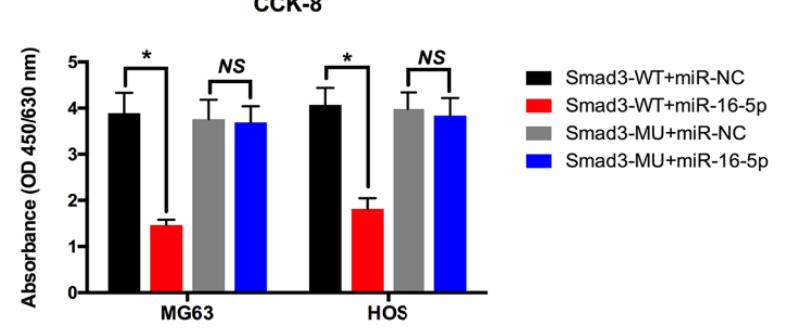

C

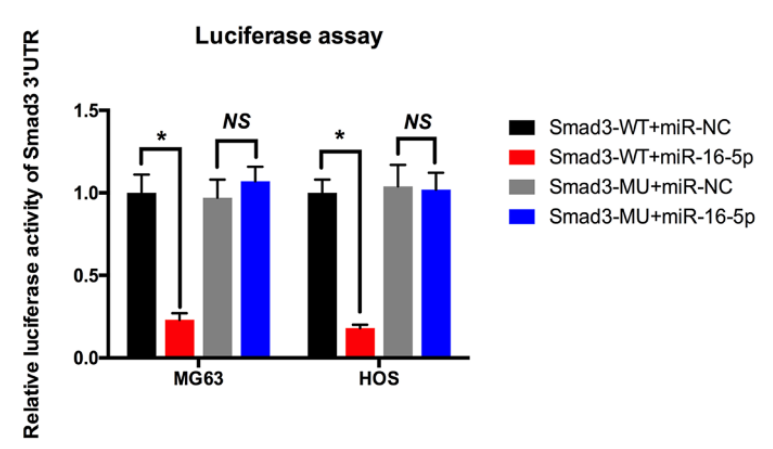

D

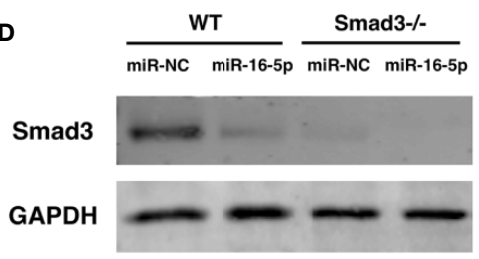

FIGURE 5 | miR-16-5p inhibits osteosarcoma cells directly targeting at Smad3. (A) A mutated Smad3 expression vector was constructed and transfected into MG63 and HOS cells. (B) the cell proliferations of wild type or mutated MG63 cells transfected with miR-NC or miR-16-5p mimics were detected using CCK-8 assays. (C) Transcription activity of Smad3 in wild type and mutated MG63 and HOS cells were measured by dual-luciferase assays. (D) Smad3 was detected in wild type and Smad3-/- cells transfected with miR-NC or miR-16-5p mimics. *indicates $p<0.05$.

et al., 2018) and gastric cancer progression (Zhang et al., 2015). Differentiation of myoblasts was regulated by miR-16-5p expression through SESN1 targeting, thereby affecting cell proliferation and apoptosis (Cai et al., 2018). However, the role of miR-16-5p in osteosarcoma is yet questionable.

In the present study, we detected the expression of miR-16-5p in human osteosarcoma and adjacent tissues and cell lines. miR-16-5p expression was significantly reduced in both osteosarcoma samples and multiple cell lines as compared with adjacent counterparts (Figure 1). To investigate the function of miR-16-5p, the mimic and inhibitor of miR-16-5p were designed and synthesized and their effects were verified by RT-PCR. Overexpression of miR-16-5p resulted in the inhibition of the proliferation, migration, and invasion of osteosarcoma cells and this effect was attenuated following transfection with miR-16-5p inhibitor (Figure 2). miR$16-5 \mathrm{p}$ is known to inhibit the growth and metastasis of chordoma by targeting the expression of Smad3 (Zhang et al., 2018). Furthermore, melatonin was shown to suppress the proliferation of gastric cancer cells by promoting the expression of miR-16-5p, which targeted Smad3 (Zhu et al., 2018). Therefore, we hypothesized that miR-16-5p downregulates Smad3 expression in osteosarcoma cells, thereby inhibiting their proliferation and enhancing their sensitivity to chemotherapy. We detected the expression of Smad3 in multiple human osteosarcoma cell lines and a human osteoblast cell line. The results of RT-PCR and immunoblot analysis demonstrated that Samd3 expression increased in osteosarcoma cells as compared with normal cells, consistent with the observations reported in human osteosarcoma samples (Figure 3). miR-16-5p mimic and inhibitor were separately transfected into MG63 and HOS cells. Overexpression of miR-16$5 \mathrm{p}$ significantly reduced $\mathrm{Smad} 3$ expression, which may be restored following transfection of cells with miR-16-5p inhibitor. Taken together, miR-16-5p suppressed the proliferation, migration, and invasion of osteosarcoma cells by regulating the expression of Smad3. Qu, et al. reported that the overexpression of miR-16-5p in breast cancer cells might inhibit cell proliferation and invasion and induce apoptosis via vascular endothelial growth factor A targeting (Qu et al., 2017).

We investigated the effect of miR-16-5p expression on the sensitivity of osteosarcoma cells to cisplatin. The therapeutic effects of cisplatin in the cells transfected with miR-16-5p mimic was obviously enhanced in a dose- and time-dependent manner. Inhibition of miR-16-5p expression resulted in a significant manifestation in the resistance of these cells to cisplatin. Incubation of osteosarcoma cells with $20 \mu \mathrm{g} / \mathrm{ml}$ of cisplatin for $48 \mathrm{~h}$ resulted in the reduction in the level of miR-16-5p with a simultaneous increase in $\mathrm{Smad} 3$ expression (Figure 4). Therefore, miR-16-5p increased the chemotherapeutic sensitivity of osteosarcoma cells to cisplatin. It was revealed that TGF- $\beta$ can up-regulate the expression of HSP27 and cisplatin resistance in human lung cancer cell through blocking the cisplatin-induced apoptosis and cell death, which characterized as the increasing of 
cell viability and decreasing of PARP and caspase 3 cleavage in the cisplatin-treated cell. Knockdown of SMAD3 attenuated the TGF$\beta$-induced HSP27 expression and enhanced the chemosensitivity of human lung cancer to cisplatin (Huang et al., 2017). In our study, miR-16-5p can down-regulate $S m a d 3$ to promote the anti-cancer effect of cisplatin on osteosarcoma. Moreover, previous research demonstrated that miR-149-5p increased the chemosensitivity of cisplatin on oral squamous cell carcinoma via inhibiting TGF- $\beta 2$, pSmad2, and p-Smad3 (Luo et al., 2019). Silencing TGF- $\beta$ or smad3 in A549/TAX (paclitaxel resistant) cells decreased the expression of cathepsin L and enhanced their sensitivity to paclitaxel (Zhao et al., 2019).

In addition, an expression vector carrying mutated or wild-type Smad3 was constructed and transfected into osteosarcoma cells. Overexpression of miR-16-5p greatly suppressed the proliferation of osteosarcoma cells. However, this suppressive effect of miR-16-5p on the proliferation of osteosarcoma cells was attenuated in the cells expressing mutated Smad3, wherein miR-16-5p was unable to recognize and bind to the mutated 3 -UTR of Smad3. Therefore, the transcription activity of Smad3 was suppressed by miR-16-5p in the cells expressing wild-type Smad3 but not mutated Smad3 (Figure 5C). The translation of Smad3 was also inhibited following overexpression of miR-16-5p (Figure 5D). We investigated the inhibitory effect of miR-16-5p on the expression of Smad3 by silencing Smad3 expression through siRNA-Smad3 transfection. As a result, the inhibitory effect of miR-16-5p on Smad3 expression was close to the effect observed with siRNASmad3 (Figure 5D). Finally, the effect of miR-16-5p on osteosarcoma in vivo was also investigated with xenograft nude model. Consequently, the tumor volume in miR-NC and miR-16$5 \mathrm{p}$ injected groups were measured and it was demonstrated that the miR-16-5p remarkably inhibited MG63 cells proliferation and induced their apoptosis in vivo. Further, the Smad3 in miR-16-5p injected group were also down-regulated in line with ex vivo results.

Taken together, we demonstrate that miR-16-5p expression is downregulated in osteosarcoma and that overexpression of miR16-5p inhibits the proliferation, migration, and invasion of osteosarcoma cells by targeting Smad3. Moreover, miR-16-5p overexpression enhances the chemotherapeutic sensitivity of osteosarcoma cells to cisplatin.

\section{REFERENCES}

Cai, B., Ma, M., Chen, B., Li, Z., Abdalla, B. A., Nie, Q., et al. (2018). MiR-16-5p targets SESN1 to regulate the p53 signaling pathway, affecting myoblast proliferation and apoptosis, and is involved in myoblast differentiation. Cell Death Dis. 9 (3), 367.

Chano, T., Avnet, S., Kusuzaki, K., Bonuccelli, G., Sonveaux, P., Rotili, D., et al. (2016). Tumour-specific metabolic adaptation to acidosis is coupled to epigenetic stability in osteosarcoma cells. Am. J. Cancer Res. 6 (4), 859-875.

Dunaeva, M., Blom, J., Thurlings, R., and Pruijn, G. J. M. (2018). Circulating serum miR-223-3p and miR-16-5p as possible biomarkers of early rheumatoid arthritis. Clin. Exp. Immunol. 193 (3), 376-385 doi: 10.1111/cei.13156

Foley, J. M., Scholten, D.J. ,. 2., Monks, N. R., Cherba, D., Monsma, D. J., Davidson, P., et al. (2015). Anoikis-resistant subpopulations of human osteosarcoma display significant chemoresistance and are sensitive to targeted epigenetic therapies predicted by expression profiling. J. Transl. Med. 13, 110.

\section{DATA AVAILABILITY STATEMENT}

All datasets generated for this study are included in the article/ Supplementary Material.

\section{ETHICS STATEMENT}

The studies involving human participants were reviewed and approved by Institutional Review Board of Tongren Hospital (No. 2019-015). The patients provided their written informed consent to participate in this study. The informed consent in accordance with the Declaration of Helsinki were signed and obtained from all donors.

\section{AUTHOR CONTRIBUTIONS}

WX and ZG initially designed the whole study. ZG, ZL, and XZ performed cellular experiments including cell culture, RT-PCR, western blot assay. Also, ZG, ZL, RH, and YX performed animal experiments. ZL, YX and WX analyzed all data. Eventually, ZG wrote and revised the manuscript under the instructions of YX and WX. All authors contributed to the article and approved the submitted version.

\section{FUNDING}

The study was supported by Excellent Young Medical Talents Training Plan of Shanghai Health Planning Commission (2018YQ46) and K. C. WONG Education Foundation (Hong Kong).

\section{SUPPLEMENTARY MATERIAL}

The Supplementary Material for this article can be found online at: https://www.frontiersin.org/articles/10.3389/fphar.2020. 01324/full\#supplementary-material

Huang, Z., Yang, C., Sun, S., Nan, Y., Lang, Z., Wang, X., et al. (2017). Heat Shock Protein 27, a Novel Regulator of Transforming Growth Factor beta Induced Resistance to Cisplatin in A549 Cell. Pharmacology 100 (5-6), 283-291.

Lei, W., Yan, C., Ya, J., Yong, D., Yujun, B., and Kai, L. (2018). MiR-199a-3p affects the multi-chemoresistance of osteosarcoma through targeting AK4. BMC Cancer 18 (1), 631 .

Li, B., Wang, Z., Wu, H., Xue, M., Lin, P., Wang, S., et al. (2018). Epigenetic Regulation of CXCL12 Plays a Critical Role in Mediating Tumor Progression and the Immune Response In Osteosarcoma. Cancer Res. 78 (14), 3938-3953.

Luo, K., He, J., Yu, D., and Acil, Y. (2019). MiR-149-5p regulates cisplatin chemosensitivity, cell growth, and metastasis of oral squamous cell carcinoma cells by targeting TGFbeta2. Int. J. Clin. Exp. Pathol. 12 (10), 3728-3739.

Meng, Y., Gao, R., Ma, J., Zhao, J., Xu, E., Wang, C., et al. (2017). MicroRNA-140$5 \mathrm{p}$ regulates osteosarcoma chemoresistance by targeting HMGN5 and autophagy. Sci. Rep. 7 (1), 416. 
Qu, Y., Liu, H., Lv, X., Liu, Y., Wang, X., Zhang, M., et al. (2017). MicroRNA-16$5 \mathrm{p}$ overexpression suppresses proliferation and invasion as well as triggers apoptosis by targeting VEGFA expression in breast carcinoma. Oncotarget 8 (42), 72400-72410.

Rinnerthaler, G., Hackl, H., Gampenrieder, S. P., Hamacher, F., Hufnagl, C., Hauser-Kronberger, C., et al. (2016). miR-16-5p Is a Stably-Expressed Housekeeping MicroRNA in Breast Cancer Tissues from Primary Tumors and from Metastatic Sites. Int. J. Mol. Sci. 17 (2), 156. doi: 10.3390/ ijms 17020156

Ritter, J., and Bielack, S. S. (2010). Osteosarcoma. Ann. Oncol. 21 (Suppl 7), vii320-vii325.

Rypens, C., Marsan, M., Van Berckelaer, C., Billiet, C., Melis, K., Lopez, S. P., et al. (2020). Inflammatory breast cancer cells are characterized by abrogated TGFbetal-dependent cell motility and SMAD3 activity. Breast Cancer Res. Treat. 180 (2), 385-395.

Saito, M., Ichikawa, J., Ando, T., Schoenecker, J. G., Ohba, T., Koyama, K., et al. (2018). Platelet-Derived TGF-beta Induces Tissue Factor Expression via the Smad3 Pathway in Osteosarcoma Cells. J. Bone Miner Res. 33 (11), 2048-2058.

Sang, S., Zhang, Z., Qin, S., Li, C., and Dong, Y. (2017). MicroRNA-16-5p Inhibits Osteoclastogenesis in Giant Cell Tumor of Bone. BioMed. Res. Int. 2017, 3173547.

Scotlandi, K., Serra, M., Nicoletti, G., Vaccari, M., Manara, M. C., Nini, G., et al. (1996). Multidrug resistance and malignancy in human osteosarcoma. Cancer Res. 56 (10), 2434-2439.

Shen, H., Wang, W., Ni, B., Zou, Q., Lu, H., and Wang, Z. (2018). Exploring the molecular mechanisms of osteosarcoma by the integrated analysis of mRNAs and miRNA microarrays. Int. J. Mol. Med. 42 (1), 21-30.

Song, B., Wang, Y., Xi, Y., Kudo, K., Bruheim, S., Botchkina, G.II, et al. (2009). Mechanism of chemoresistance mediated by miR-140 in human osteosarcoma and colon cancer cells. Oncogene 28 (46), 4065-4074.

Song, Y. D., Li, D. D., Guan, Y., Wang, Y. L., and Zheng, J. (2017). miR-214 modulates cisplatin sensitivity of osteosarcoma cells through regulation of anaerobic glycolysis. Cell Mol. Biol. (Noisy-le-grand) 63 (9), 75-79.

Tang, P. M., Zhou, S., Meng, X. M., Wang, Q. M., Li, C. J., Lian, G. Y., et al. (2017). Smad3 promotes cancer progression by inhibiting E4BP4-mediated NK cell development. Nat. Commun. 8, 14677.
Wang, S. N., Luo, S., Liu, C., Piao, Z., Gou, W., Wang, Y., et al. (2017). miR-491 Inhibits Osteosarcoma Lung Metastasis and Chemoresistance by Targeting alphaB-crystallin. Mol. Ther. 25 (9), 2140-2149.

Xie, L., Yao, Z., Zhang, Y., Li, D., Hu, F., Liao, Y., et al. (2018). Deep RNA sequencing reveals the dynamic regulation of miRNA, IncRNAs, and mRNAs in osteosarcoma tumorigenesis and pulmonary metastasis. Cell Death Dis. 9 (7), 772 .

Yang, J., and Zhang, W. (2013). New molecular insights into osteosarcoma targeted therapy. Curr. Opin. Oncol. 25 (4), 398-406.

Zhang, J., Song, Y., Zhang, C., Zhi, X., Fu, H., Ma, Y., et al. (2015). Circulating MiR-16-5p and MiR-19b-3p as Two Novel Potential Biomarkers to Indicate Progression of Gastric Cancer. Theranostics 5 (7), 733-745.

Zhang, L., Pan, L., Xiang, B., Zhu, H., Wu, Y., Chen, M., et al. (2016). Potential role of exosome-associated microRNA panels and in vivo environment to predict drug resistance for patients with multiple myeloma. Oncotarget 7 (21), 3087630891.

Zhang, H., Yang, K., Ren, T., Huang, Y., Tang, X., and Guo, W. (2018). miR-16-5p inhibits chordoma cell proliferation, invasion and metastasis by targeting Smad3. Cell Death Dis. 9 (6), 680.

Zhao, Y., Shen, X., Zhu, Y., Wang, A., Xiong, Y., Wang, L., et al. (2019). Cathepsin L-mediated resistance of paclitaxel and cisplatin is mediated by distinct regulatory mechanisms. J. Exp. Clin. Cancer Res. 38 (1), 333.

Zhu, C., Huang, Q., and Zhu, H. (2018). Melatonin Inhibits the Proliferation of Gastric Cancer Cells Through Regulating the miR-16-5p-Smad3 Pathway. DNA Cell Biol. 37 (3), 244-252.

Conflict of Interest: The authors declare that the research was conducted in the absence of any commercial or financial relationships that could be construed as a potential conflict of interest.

Copyright (c) $2020 \mathrm{Gu}, \mathrm{Li}, \mathrm{Xu}, \mathrm{Zhu}, \mathrm{Hu}, \mathrm{Xue}$ and $\mathrm{Xu}$. This is an open-access article distributed under the terms of the Creative Commons Attribution License (CC BY). The use, distribution or reproduction in other forums is permitted, provided the original author(s) and the copyright owner(s) are credited and that the original publication in this journal is cited, in accordance with accepted academic practice. No use, distribution or reproduction is permitted which does not comply with these terms. 